\title{
THE EXPRESSION OF DETERMINATION: SIMILARITIES BETWEEN ANGER AND APPROACH-RELATED POSITIVE AFFECT
}

\author{
A Thesis \\ by \\ CINDY HARMON-JONES
}

\begin{abstract}
Submitted to the Office of Graduate Studies of
Texas A\&M University

in partial fulfillment of the requirements for the degree of

MASTER OF SCIENCE
\end{abstract}

December 2009

Major Subject: Psychology 


\title{
THE EXPRESSION OF DETERMINATION: SIMILARITIES BETWEEN \\ ANGER AND APPROACH-RELATED POSITIVE AFFECT
}

\author{
A Thesis \\ by \\ CINDY HARMON-JONES
}

\begin{abstract}
Submitted to the Office of Graduate Studies of Texas A\&M University

in partial fulfillment of the requirements for the degree of

MASTER OF SCIENCE
\end{abstract}

Approved by:

Co-Chairs of Committee, Brandon J. Schmeichel Gerianne Alexander

Committee Member, $\quad$ Karen Winterich

Head of Department, Leslie Morey

December 2009

Major Subject: Psychology 


\begin{abstract}
The Expression of Determination: Similarities Between

Anger and Approach-Related Positive Affect. (December 2009)

Cindy Harmon-Jones, B.S., Excelsior College

Co-Chairs of Advisory Committee: Dr. Brandon J. Schmeichel

Dr. Gerianne Alexander
\end{abstract}

This study examines the valence and motivational direction components of affect using facial expressions of determination, anger and joy. Determination is a positive, approach-related emotion; anger is a negative, approach-related emotion; and joy is a positive, low-approach emotion. Thus, determination and anger share a motivational direction, but determination and joy share a valence. Participants created facial expressions intended to express joy, anger, fear, sadness, disgust and determination. Naïve judges attempted to identify these expressions. Correct identifications of intended determination expressions were positively correlated with misidentifications of the expressions as anger, suggesting that determination is perceived as more similar to anger than to joy. This emphasizes the importance of the motivational component of emotion, as distinct from the valence of emotion. 


\section{ACKNOWLEDGEMENTS}

I would like to thank my committee chair, Dr. Brandon Schmeichel, for supporting me in pursuing this research. I'd also like to thank my committee co-chair, Dr. Gerianne Alexander, and committee member, Dr. Karen Winterich, for their guidance and support.

My gratitude also goes to my husband, Dr. Eddie Harmon-Jones, for his methodological and theoretical expertise. Eddie, your passion for psychological science is infectious. You are an inspiration. Finally, thanks to my children, Sylvia and Leon, for their patience in listening to endless conversations about psychology, in the car and at the dinner table. 
NOMENCLATURE

NA

PA

PANAS
Negative Affect

Positive Affect

Positive and Negative Affect Schedule 
TABLE OF CONTENTS

Page

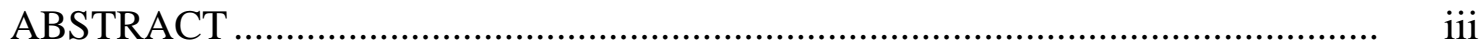

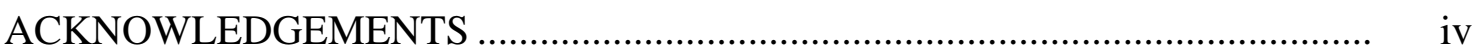

NOMENCLATURE ........................................................................................ v

TABLE OF CONTENTS ……………………….................................... vi

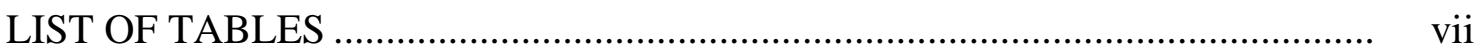

INTRODUCTION: ANGER RELATES TO POSITIVE AFFECT ……………….... 1

A Prominent Model of Affect ................................................................ 3

Anger: An Approach-Related Negative Affect..................................... 5

Anger and PA: Positively Related during Anger Episodes................... 6

Facial Expressions as a Means to Study Emotion.................................. 7

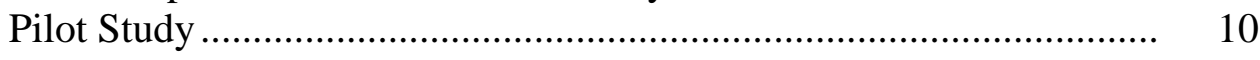

MASTER'S THESIS STUDY ……………………..................................... 12

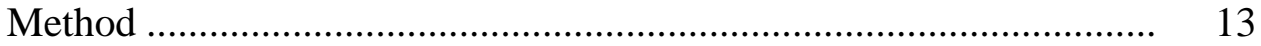

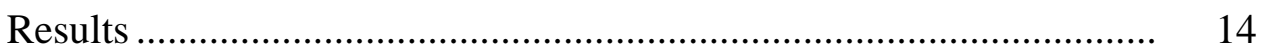

SUMMARY AND CONCLUSIONS............................................................. 18

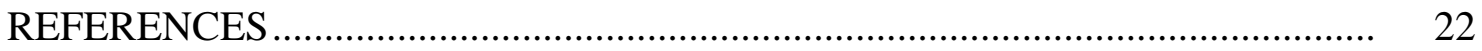

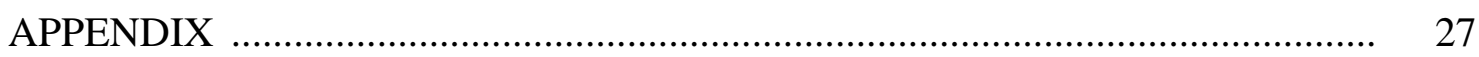

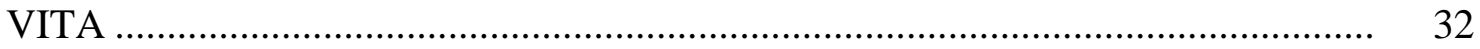




\section{LIST OF TABLES}

TABLE

1 Correlations for Intended Determination Expressions:

Correct and Mis-Identifications.

2 Percentages of Identifications of Emotion Expressions for Individual Expressers ....

3 Correlations for Emotion Identifications and

Individual Differences Measures 


\section{INTRODUCTION:}

\section{ANGER RELATES TO POSITIVE AFFECT}

The facial expression of determination may provide a novel way of examining the motivational component of emotion. Determination is an affect that is classified as positive by a prominent model of emotion (Watson \& Tellegen, 1985; Watson, Clark, \& Tellegen, 1988). However, ratings of determination increase during the experience of anger (Harmon-Jones, Vaughn-Scott, Mohr, Sigelman, \& Harmon-Jones, 2004; HarmonJones, Harmon-Jones, Abramson, \& Peterson, 2009). Harmon-Jones and colleagues suggested that determination increases during anger because determination and anger are related to high approach motivation. In the current study, we examine the facial expression of determination, and whether it is more similar to expressions of positive affect (regardless of motivational intensity) or to approach-related affect (regardless of valence).

The research cited above relied on self-reports to assess emotion. However, emotions are processes that also include experiential, physiological, behavioral and expressive components. The current study uses facial expressions to examine similarities and differences between affects that vary in their motivational directions and valences, specifically determination, anger, and joy.

This thesis follows the style of Journal of Personality and Social Psychology. 
Prominent models of affect state that the basic structure of emotions is organized around two dimensions, arousal and valence (e.g., Lang, 1995; Russell, 1980; Watson \& Tellegen, 1985). Arousal varies from low to high and valence varies from negative to positive. Some dimensional models also postulate a relationship between affective valence and motivational direction, such that positive affect is directly related to approach motivation, while negative affect is directly related to withdrawal motivation (Watson, 2000). However, Harmon-Jones and colleagues have shown that motivational direction is separable from valence, and that negative affect can be associated with approach motivation (Harmon-Jones \& Allen, 1998; Harmon-Jones \& Sigelman, 2001; Harmon-Jones, Sigelman, Bohlig, \& Harmon-Jones, 2003).

The current study examines the motivational and valence dimensions of emotions via the relationship between facial expressions of determination, anger and joy. Determination is a positive affect that is high in approach motivation (Watson et al., 1988). Anger is a negative affect that is high in approach motivation (Carver, 2004; Harmon-Jones, 2003; Harmon-Jones \& Allen, 1998). Because joy is a positive affect that is produced when a desired outcome is achieved, it is a positive affect that is low in approach motivation (Gable \& Harmon-Jones, 2008). Thus, determination and anger share a common motivational direction, and determination and joy share a common valence. If valence is the more important factor influencing the perception of facial expressions, then determination should be perceived as similar to joy. However, if motivation is the more important factor influencing the perception of facial expressions, then determination should be perceived as similar to anger. 


\section{A Prominent Model of Affect}

Watson (2000) suggested that positive affect (PA) is equivalent to approach motivation. He wrote, “... a growing body of evidence has suggested that negative mood experience is part of a larger Behavioral Inhibition System (BIS), whereas positive mood experience is linked to what has variously been called the Behavioral Activation System, Behavioral Engagement System, or Behavioral Facilitation System.” (Watson, 2000, p. 22-23). Other theories of emotion propose similar ideas (e.g., Cacioppo, Gardner, \& Berntson, 1999; Gray, 1990; Lang, 1995; Lang, Bradley, \& Cuthbert, 1990, 1992, 1998; Larsen, McGraw, \& Cacioppo, 2001).

Based on this model, Watson and colleagues (1988) created the Positive and Negative Affect Schedule (PANAS, Watson \& Clark, 1994). This scale has been widely used in psychological research to assess emotion. In 1999, its scales were renamed positive activation (PA) and negative activation (NA) to convey the activated nature of these dimensions (Watson, 1999). According to Watson et al. (1988), the positive items measure a dimension defined as activation plus pleasantness, and the negative items measure a dimension defined as activation plus unpleasantness. "High PA is a state of high energy, full concentration, and pleasurable engagement, whereas low PA is characterized by sadness and lethargy. In contrast, Negative Affect (NA) is a general dimension of subjective distress and unpleasurable engagement that subsumes a variety of aversive mood states, including anger, contempt, disgust, guilt, fear, and nervousness,...” (p. 1063). Gray and Watson more recently (2007, p. 173) wrote, 
"Positive Affect is composed of positively valenced mood states, including enthusiasm, energy, interest, pleasure, confidence, and feelings of affiliation... state positive affect reflects an individual's short-term, often context-specific, experience of positive emotions such as confidence or joy.”

Affective valence may correlate with motivational direction in many cases. For example, research on emotion has frequently examined affects, such as fear, which relate to both negative valence and withdrawal motivation. Affects that violate the hypothesized relationship between motivational direction and valence, such as anger, have been relatively neglected. Moreover, the methods used to identify the emotion words used on the PANAS may have obscured more complex relationships between emotions, their valences, and their associated motivations.

The items on the PANAS were selected from a large list of affect terms by means of principal components analysis (Watson et al., 1988). Items were selected if they had a substantial loading on one factor and a near-zero loading on the other. This resulted in the following items for the PA scale: active, alert, attentive, determined, enthusiastic, excited, inspired, interested, proud, and strong; and the following items for the NA scale: afraid, scared, nervous, jittery, irritable, hostile, guilty, ashamed, upset, and distressed. Researchers are sometimes surprised that certain "basic" emotion words are not found in the PANAS, including joyful (and synonyms such as happy), and angry (and synonyms such as mad). 


\section{Anger: An Approach-Related Negative Affect}

A body of research suggests that eliminating affects (such as anger) that violate the hypothetical direct relationship between valence and motivation may have produced a misleading picture of the structure of emotion. Anger violates the postulate of the valence model of emotion that approach motivation is always positively valenced. Anger is a negative emotion, yet theorists have long suggested that anger evokes behavioral approach tendencies (e.g., Darwin, 1872; Plutchik, 1980). Animal behavior theorists note that anger-induced irritable or offensive aggression is associated with attack and no attempts to escape, whereas fear-induced defensive aggression is associated with attempts to escape and attack only if escape is impossible (Blanchard \& Blanchard, 1984). Developmental psychologists have also found that angry facial expressions are associated with approach motivation (Lewis et al., 1990; Lewis et al., 1992).

Individual's trait tendencies toward approach and withdrawal, as measured by Carver and White’s (1994) behavioral inhibition/behavioral activation sensitivity (BIS/BAS) scales, are also related to anger. In two studies, Harmon-Jones (2003) showed that trait approach motivation, as measured by the BAS scale, related positively to trait anger, as assessed by the Buss and Perry (1992) anger subscale of the Aggression Questionnaire. In a second study, BAS was also related to physical aggression. Smits and Kuppens (2005) found that the tendency to express one’s anger outwardly (assessed by questions such as, "I tell my friends openly when I disagree with them.”) related directly to BAS scores, whereas the tendency to express one’s anger inwardly related 
directly to BIS scores. Carver (2004) also found that self-reported frustration and anger in response to situational elicitors was related to greater trait BAS. Thus, longstanding theories of emotion, animal research, developmental research, and research assessing both state and trait approach motivation support the idea that anger is a negative affect associated with approach tendencies.

Anger and PA: Positively Related during Anger Episodes

Anger and PA may also be related, due to their common association with approach motivation. Harmon-Jones, Vaughn-Scott, Mohr, Sigelman, and Harmon-Jones (2004) found that both self-reported anger and PA increased following an insult. In this experiment, anger was induced by giving participants insulting feedback, and then selfreported emotion was measured. Anger and PA were greater in the insult condition than in the no-insult condition. Harmon-Jones, Harmon-Jones, Abramson, and Peterson (2009) also showed that an anger-inducing event increased self-reports of both anger and PA. Moreover, anger and PA were directly associated with each other following an anger-evoking event. These results suggest that the PA scale assesses approach motivation, rather than purely positive activation.

In a third study reported in Harmon-Jones et al. (2009), participants remembered a time when they were really angry and reported how they felt during that experience. Anger was measured using the items hostile, angry, irritated, agitated, frustrated, furious, enraged, and mad. Happiness was measured using the words glad, content, 
pleasant, pleased, tranquil, well, calm, good mood, joyful, satisfied, and happy. PA and NA were measured using the PANAS (Watson et al., 1988). PA correlated directly with both anger and happiness, while anger correlated inversely with happiness. In this study, attitudes toward anger were also assessed, and the correlation between anger and PA was not due to a positive attitude toward anger. Thus, anger and PA were associated during the self-reported recall of anger episodes.

\section{Facial Expressions as a Means to Study Emotion}

The above research used self-reported emotions as a way to study affect. However, emotions are phenomena that involve multiple components, and self-reports alone may not provide a full understanding of their structure and functions. Another prominent component of affect is the facial and bodily expression of emotions (Darwin, 1872; Ekman, 1992). Examination of the connections between anger and some forms of positive affect at the expressive level of analysis is important theoretically and can provide a different means of examining affect. Based on the self-report research summarized above, I predict that facial expressions of approach-related positive affects may be perceptually confused with anger.

The past research reviewed above showed that an angering situation increases ratings on the PANAS items that are intended to measure positive affect. These results suggest that the PA items measure both positive valence and approach motivation-even when the approach motivation is negative. That is, PA correlates directly with both 
happiness and anger, but happiness and anger are inversely correlated. The current study will compare the facial expressions associated with an approach-motivated, positive emotion, and expressions of anger, an approach-motivated, negative emotion, as well as joy, a low-approach, positive emotion. The research reviewed above revealed that determined is an item from the PA scale that is consistently and robustly increased by anger manipulations (Harmon-Jones et al., 2004; Harmon-Jones et al., 2009), so the facial expression of determination will be examined in this study.

Watson and colleagues (2000) situated PA items such as determination at the activated, positive pole in their model of emotion. In contrast, early facial expression researchers referred to an anger/determination complex that was situated at the unpleasant pole of their emotion scale (Woodworth \& Schlosberg, 1938/1954; Schlosberg, 1941). Schlosberg (1941) used a sorting method to classify facial expressions of emotion. Based on these judgments, he created a circular scale with two axes. He labeled one of these axes pleasant vs. unpleasant. The other axis was labeled attention (or acceptance) vs. rejection. Schlosberg found that anger/determination expressions anchored the unpleasant axis of the scale. Levy and Schlosberg (1960) replicated these results using a different set of facial expression photographs.

Facial expressions of both joy and anger, as well as the other basic emotion expressions (fear, disgust, and sadness), are reliably recognizable in Western and nonWestern cultures (Ekman, \& Friesen, 1971; Ekman, 1992). Research has shown that emotions beyond the basic emotions have identifiable facial expressions as well, including contempt (Matsumoto \& Ekman, 2004) and pride (Tracy \& Robins, 2004; 
Tracy, Robins, \& Lagattuta, 2005). The current research will examine whether the facial expression of determination is identifiable by participants, in addition to examining its similarities to joy and anger.

Personality traits may also relate to individuals' abilities to express emotion with the face. Malatesta, Fiore, and Messina (1987) found that the facial characteristics of elderly participants, as judged by naïve and trained raters, were related to the expressers' personalities. The researchers photographed 14 senior citizens while they created emotional (angry, sad, happy, and fearful) and neutral facial expressions. Then, judges identified the emotion expressed in each photograph. The researchers then examined the patterns of misidentification of facial poses that were intended to be neutral. They found statistically significant correlations between misidentifications of anger, sadness, contempt, and guilt, and the subjects' self-ratings of these emotions on the Differential Emotions Scale (Izard, 1972). The researchers suggested that a lifetime of habitually experiencing these emotions may have etched them onto the faces of their participants. However, the researchers also noted that it is not clear whether these effects are limited to older persons. Thus, it is possible that trait approach motivation and trait anger could relate to the ability to create voluntary expressions of determination and anger, similar to the relationship between self-reported trait approach motivation and trait anger (Harmon-Jones, 2003). 
Pilot Study

Preliminary research examined the facial expression of determination compared to other facial expressions of emotion. The prediction was that the facial expression of determination would be perceived as more similar to anger than to joy.

Method

Four individuals who could make easily identifiable facial expressions of the basic emotions were brought to the lab and photographed while making the basic emotion facial expressions (joy, anger, sadness, fear, and disgust), determination, and a neutral expression. To create each expression, the participants were told, "Please make expressions to communicate the emotion as strongly as possible. Try to make an expression so that absolutely anyone would be able to recognize what emotion you are communicating.”

Following this, arrays of the facial expression photographs were given to 10 participants who were naïve to the hypothesis. Participants were asked to indicate what each expression was displaying, from the following eight options: anger, sad, fear, disgust, joy, determined, neutral, or none of the above (as recommended by Matsumoto \& Ekman, 2004).

Results

Intended determination expressions were correctly identified as determined significantly better than chance, $\chi^{2}(7)=108.00, \mathrm{p}<.001$. Intended determination 
expressions were misidentified as anger significantly more often than as joy, $\chi^{2}(6)=$ $18.00, \mathrm{p}<.001$.

\section{Discussion}

The results of the pilot study suggest that the affect of determination has a facial expression that can be identified by perceivers. The results also support the hypothesis that facial expressions of determination are more likely to be confused with anger than with joy by perceivers. This suggests that expressions of anger and determination are perceived as similar, even though determination is classified as positive while anger is classified as negative. These preliminary results add to evidence that PA, as measured by the PANAS, is a measure of approach motivation and does not uniquely measure positive valence. 


\section{MASTER'S THESIS STUDY}

The current study replicates and extends the pilot study described above, using a larger sample both of emotion expressers and judges. Self-reported traits of anger and approach motivation are also assessed, to examine the relationship between traits and the ability to voluntarily create facial expressions. Buss and Perry’s (1992) Aggression Questionnaire is used to assess trait anger, and the BIS/BAS scale (Carver \& White, 1994) is used to assess trait approach motivation.

The current study also improves upon the pilot study by presenting the photographs one at a time, instead of in an array. Presenting photographs in an array may assist participants in identifying emotional expressions by allowing them to rely on the process of elimination.

Much research on facial expressions has focused on which emotions are universally identifiable. The intention of the current study is not to examine whether determination is universally expressed or universally recognized. In fact, we expected that many naïve expressers might not be capable of creating a recognizable determination expression. Instead, the hypothesis is that when an intended determination expression is identifiable as determination, it will be similar in appearance to anger. Furthermore, when an intended determination expression is identifiable, it will be dissimilar in appearance from joy. 
Method

Participants

The participants were 33 introductory psychology undergraduates at Texas A \&

M University (11 male, 22 female) who answered “yes” to three pre-screening questions: 1. “Are you good at communicating emotions with your face?”; 2. "Would you like to participate in an experiment where you would be photographed while making emotional expressions with your face?”; 3. "Would you be willing for photographs of your face, making emotional expressions, to be shown to participants in other studies?” The participants were volunteers who participated in exchange for course credit.

Procedure

Participants were brought to the lab individually. They were photographed while making the basic emotion facial expressions (joy, anger, sadness, fear, and disgust), determination, and a neutral expression. To create each expression, the participants were told, "Please express the emotion as clearly as you can. Try to make an expression so that absolutely anyone would be able to recognize what emotion you are communicating.” The rationale for asking participants to naturalistically make the facial expressions, instead of instructing them which muscles to contract, is to identify those participants who can make recognizable basic emotion expressions and to examine the relationship of this ability to the ability to create recognizable determination expressions.

Participants then completed personality questionnaires assessing traits that may be associated with the ability to create the facial expressions of interest: Buss and 
Perry’s (1992) Aggression Questionnaire, and the BIS/BAS scale (Carver \& White, 1994).

These picture stimuli were presented to a separate group of naïve participants by computer using www.surveymonkey.com. Photographs were presented individually. Judgment Tasks

The naïve judges were 462 introductory psychology undergraduates from Texas A \& M University, who participated in exchange for course credit. Judges were asked to identify the emotion expressed in each photograph, selecting from a list that included “neutral,” “joy,” “anger,” “sad,” "fear,” “disgust,” “determined” and “none of these,” as recommended by Matsumoto and Ekman (2004).

\section{Data Processing}

The percentage of correct identifications of each facial expression was calculated. The percentage of misidentifications of each expression was calculated by dividing the number of incorrect identifications as each specific emotion by the total number of incorrect identifications. For example, for a photograph that is intended to express joy, the percent misidentifications as anger is the number of identifications as anger divided by the total number of misidentifications for that photograph, multiplied by 100 .

\section{Results}

We predicted that intended determination expressions that were reliably identified as determination would appear similar to anger. Results showed that correct 
identifications of intended determination expressions were positively related to the percentage of misidentifications of the same expression as anger, $r(31)=.37, p=.036$, as shown in Table 1. Correct identifications of intended determination expressions were not significantly related to misidentifications as neutral, joy, sadness, disgust, fear or none of these. These results support the primary hypothesis.

The percentage of judges selecting the correct label for each intended joy, anger and determination expression was computed, as shown in Table 2. The percentages of misidentifications as joy, anger, and determination were also computed. Of 33 intended determination expressions, 12 (36\%) were identified as neutral as the modal response, 7 (21\%) were identified as determined as the modal response, $6(18 \%)$ were identified as anger as the modal response, 2 (6\%) were identified as joy, 2 (6\%) were identified as disgust, and 2 (6\%) were identified as none of these, as the modal response, 1 (3\%) was identified as sadness as the modal response, and none $(0 \%)$ were identified as fear as the modal response. Chi-square tests compared the percentage of judges correctly identifying the expressions, with expected frequencies set at one-eighth (i.e., chance). The seven expressions that were correctly identified as determination as the modal response all had recognition levels much higher than chance, according to chi-squared tests ( $d f=8, N=462$, for all chi-square tests), $\chi^{2}=238.03, p<.001, \chi^{2}=77.83, p<$ $.001, \chi^{2}=72.22, p<.001, \chi^{2}=180.41, p<.001, \chi^{2}=119.11, p<.001, \chi^{2}=72.38, p<$ $.001, \chi^{2}=137.52, p<.001$, respectively, by order of data in Table 2 .

The motivational direction model did not suggest an a priori prediction regarding the relationship of sex of expresser and the ability to create identifiable facial 
expressions, so we explored this relationship with a 2 (sex) x 3 (facial expression: determination, anger, and joy) mixed ANOVA predicting correct identifications. Facial expression generated a significant main effect, $F(2,62)=182.20, p<.001$. This was the result of greater correct identifications for joy expressions, followed by anger, and then determination. The sex of expresser main effect and the facial expression $\mathrm{x}$ sex interactions were not significant, $F$ 's $<1.0$, $p$ 's $>$.36.

We predicted that participants who were able to create identifiable determination expressions would have personality characteristics associated with anger and approach motivation. At the individual differences level, correct identifications of intended determination expressions were marginally related to the expresser's trait anger, $r(31)=$ $.30, p=.09$, and trait physical aggression, $r(31)=.32, p=.06$, as shown in Table 3 . Although these correlations are marginally significant, it is important to note that they are in the predicted direction, and are opposite to the direction that would be predicted by the valence model (i.e., the valence model would predict a significant negative correlation).

We predicted that participants who were able to make identifiable anger expressions would also have personality traits associated directly with approach motivation, and perhaps inversely with BIS. At the individual differences level, correct identifications of intended anger expressions were related to the expresser's BAS reward responsiveness, $r(31)=.44, p=.01$, and BAS fun-seeking, $r(31)=.35, p=.05$, and marginally related to BAS drive, $r(31)=.30, p=.09$, and marginally negatively related to BIS, $r(31)=-.32, p=.07$, as shown in Table 3 . 
We predicted that participants who were able to make identifiable joy expressions would not have personality traits associated with anger or approach motivation. At the individual differences level, correct identifications of intended joy expressions were significantly negatively related to expressers’ trait physical aggression, $r(31)=-.48, p=.004$, were marginally negatively related to trait verbal aggression, $r$ $(31)=-.33, p=.06$, and were marginally positively related to BIS, $r(31)=.30, p=.09$, as shown in Table 3. The relationship of correct identifications of joy expressions and trait physical aggression was in the opposite direction from the relationship of correct identifications of determination expressions and trait physical aggression. 


\section{SUMMARY AND CONCLUSIONS}

This study advances the understanding of approach-oriented emotions by examining the facial expression of determination-a positive, approach-related emotion. This study compared facial expressions intended to express determination with facial expressions intended to express anger and joy. Determination and joy are similar in that they share a positive valence. Determination and anger share an association with approach motivation, but differ in valence. Determination is used as an exemplar of activated positive affect on the PANAS (Watson et al., 1988), a commonly used instrument for measuring emotion. However, determination has previously been identified as similar to anger according to early emotional expression theories (Woodworth \& Schlosberg, 1938/1954; Schlosberg, 1941).

As predicted, the results of the current studies showed that the expression of determination is perceived as similar to the expression of anger. In a pilot study, four expressers created facial expressions of the basic emotions, plus determination. An array of these photographs was given to 10 naïve judges. Expressions intended to express determination were confused with anger significantly more often than with joy.

The current study replicated and extended the pilot study. In the current study, 33 naïve expressers were photographed while creating the basic emotions plus determination. Of these 33 participants, only seven created determination expressions that were reliably identified at greater than chance levels. Thus, many naïve participants were not able to create recognizable determination expressions. This result was not 
unexpected. The intent of this research was not to demonstrate that naïve participants are reliably able to create recognizable expressions that communicate determination. Rather, our prediction was that, to the extent that an expression is recognizable as determination, it will appear similar to anger. This prediction was supported. Correct identifications of intended determination expressions were positively related to misidentifications as anger. In contrast, correct identifications of intended determination expressions were not significantly related to misidentifications as neutral, joy, sadness, disgust, fear or none of these.

Further support to our hypothesis was provided by individual differences measures. We predicted that the personality characteristics of trait anger and trait approach motivation would relate to the ability to express both determination and anger. In contrast, we predicted that these characteristics would not relate positively to the ability to express joy. The results provided some support for these hypotheses. The ability to create identifiable determination expressions was marginally related to trait anger and trait physical aggression. The ability to create identifiable anger expressions was significantly related to approach motivation as measured by BAS reward responsiveness and BAS fun-seeking, marginally related to BAS drive, and was marginally negatively related to BIS. In contrast, the ability to create identifiable joy expressions was significantly negatively related to trait physical aggression, and marginally negatively related to trait verbal aggression and trait anger, and marginally positively related to BIS. Thus, approach-related personality characteristics related 
positively to the ability to express anger and determination, while these characteristics related negatively to the ability to express joy.

The results of this study emphasize the importance of the motivational component of emotion, as distinguished from the valence of emotion. The valence of emotion has been prominent in most past research on self-reported moods (which have often been evaluated under non-emotional circumstances), and consequently, the valence of emotion often serves as a guiding principle in more behavioral and physiological research on emotion. However, the motivational component of emotion may be more prominent during the perception of facial expressions, as suggested by early facial expression research (Schlosberg, 1941; Woodworth \& Schlosberg, 1938/1954) and as suggested by the results of the current study. It is possible that the behavioral intentions of the expresser may be highly salient to perceivers of facial expressions, with behavioral intentions related more closely to motivation than to valence. In contrast, the valence of emotion may be highly salient to the individual who is self-reporting an emotion, while the motivational components are not as consciously accessible.

The results also enhance our understanding of the relationship between personality traits and the ability to express emotions. Some previous research suggests that personality traits influence judges' perception of emotion in intended neutral facial expressions, at least in elderly expressers (Malatesta et al., 1987). The current study suggests that personality traits relate to younger individuals' abilities to create identifiable facial expressions. Specifically, the current study showed a relationship between trait BAS and individuals' abilities to express anger. The current study also 
suggested a possible relationship between trait anger and individuals' abilities to express determination, although this did not reach statistical significance. The current study also showed a negative relationship between trait anger and the ability to express joy. Future research should examine other personality correlates of the ability to express specific emotions. 


\section{REFERENCES}

Blanchard, D. C., \& Blanchard, R. J. (1984). Affect and aggression: An animal model applied to human behavior. In: R. J. Blanchard, \& D. C. Blanchard (Eds.), Advances in the study of aggression (pp. 1-62), New York: Academic Press.

Buss, A. H., \& Perry, M. (1992). The aggression questionnaire. Journal of Personality and Social Psychology, 63, 452-459.

Cacioppo, J. T., Gardner, W. L., \& Berntson, G. G. (1999). The affect system has parallel and integrative processing components: Form follows function. Journal of Personality and Social Psychology, 76, 839-855.

Carver, C. S. (2004). Negative affects deriving from the behavioral approach system. Emotion, 4, 3-22.

Carver, C. S., \& White, T. L. (1994). Behavioral inhibition, behavioral activation, and affective responses to impending reward and punishment: The BIS/BAS scales. Journal of Personality and Social Psychology, 67, 319-333.

Darwin, C. (1872/1965). The expressions of the emotions in man and animals. New York: Oxford University Press.

Ekman, P. (1992) An argument for basic emotions. Cognition and Emotion, 6, 169-200.

Ekman, P., \& Friesen, W. (1971). Constants across culture in the face and emotion. Journal of Personality and Social Psychology, 17, 124-129. 
Gable, P. A., \& Harmon-Jones, E. (2008). Approach-motivated positive affect reduces breadth of attention. Psychological Science, 19, 476-482.

Gray, E. K., \& Watson, D. (2007). Assessing positive and negative affect via self-report. In J. A. Coan \& J. J. B. Allen (Eds.), Handbook of emotional elicitation and assessment (pp. 171-183). New York: Oxford University Press.

Gray, J. A. (1990). Brain systems that mediate both emotion and cognition. Cognition \& Emotion, 4, 269-288.

Harmon-Jones, E. (2003). Anger and the behavioural approach system. Personality and Individual Differences, 35, 995-1005.

Harmon-Jones, E. (2004). On the relationship of anterior brain activity and anger: Examining the role of attitude toward anger. Cognition and Emotion, 18, 337361.

Harmon-Jones, E., \& Allen, J. J. B. (1998). Anger and prefrontal brain activity: EEG asymmetry consistent with approach motivation despite negative affective valence. Journal of Personality and Social Psychology, 74, 1310-1316.

Harmon-Jones, E., Harmon-Jones, C., Abramson, L. Y., \& Peterson, C. K. (2009). PANAS positive activation is associated with anger. Emotion, 9, 183-196.

Harmon-Jones, E., \& Sigelman, J. (2001). State anger and prefrontal brain activity: Evidence that insult-related relative left prefrontal activation is associated with experienced anger and aggression. Journal of Personality and Social Psychology, 80, 797-803. 
Harmon-Jones, E., Sigelman, J. D., Bohlig, A., \& Harmon-Jones, C. (2003). Anger, coping, and frontal cortical activity: The effect of coping potential on angerinduced left frontal activity. Cognition and Emotion, 17, 1-24.

Harmon-Jones, E., Vaughn-Scott, K., Mohr, S., Sigelman, J., \& Harmon-Jones, C. (2004). The effect of manipulated sympathy and anger on left and right frontal cortical activity. Emotion, 4, 95-101.

Izard, C. (1972). Patterns of emotions: A new analysis of anxiety and depression. New York: Academic Press.

Lang, P. J. (1995). The emotion probe: Studies of motivation and attention. American Psychologist, 50, 372-385.

Lang, P. J., Bradley, M. M., \& Cuthbert, B. N. (1990). Emotion, attention, and the startle reflex. Psychological Review, 97, 377-395.

Lang, P. J., Bradley, M. M., \& Cuthbert, B. N. (1992). A motivational analysis of emotion - reflex cortex connections. Psychological Science, 3, 44-49.

Lang, P. J., Bradley, M. M., \& Cuthbert, B. N. (1998). Emotion, motivation, and anxiety: Brain mechanisms and psychophysiology. Biological Psychiatry, 44, $1248-1263$.

Larsen, J. T., McGraw, A. P., \& Cacioppo, J. T. (2001). Can people feel happy and sad at the same time? Journal of Personality and Social Psychology, 81, 684-696.

Levy, N., \& Schlosberg, H. (1960). Woodworth scale values of the Lightfoot pictures of facial expression. Journal of Experimental Psychology, 60, 121-125. 
Lewis, M., Alessandri, S. M., \& Sullivan, M. W. (1990). Violation of expectancy, loss of control, and anger expressions in young infants. Developmental Psychology, 26, 745-751.

Lewis, M., Sullivan, M. W., Ramsay, D. S., \& Alessandri, S. M. (1992). Individual differences in anger and sad expressions during extinction: Antecedents and consequences. Infant Behavior \& Development, 15, 443-452.

Malatesta, C. Z., Fiore, M. J., \& Messina, J. J. (1987). Affect, personality, and facial expressive characteristics of older people. Psychology and Aging, 2, 64-69.

Matsumoto, D. \& Ekman, P. (2004). The relationship among expressions, labels, and descriptions of contempt. Journal of Personality and Social Psychology, 87, 529540.

Plutchik, R. (1980). Emotion: A psychoevolutionary synthesis. New York: Harpercollins College Division.

Russell, J. A. (1980). A circumplex model of affect. Journal of Personality and Social Psychology, 39, 1161-1178.

Schlosberg, H. (1941). A scale for the judgment of facial expressions. Journal of Experimental Psychology, 29, 497-510.

Smits, D. J. M., \& Kuppens, P. (2005). The relations between anger, coping with anger, and aggression, and the BIS/BAS system. Personality and Individual Differences, 39, 783-793.

Tracy, J. L., \& Robins, R. W. (2004). Show your pride: Evidence for a discrete emotion expression. Psychological Science, 15, 194-197. 
Tracy, J. L., Robins, R. W., \& Lagattuta, K. H. (2005). Can children recognize pride? Emotion, 5, 251-257.

Watson, D. (2000). Mood and temperament. New York: Guilford Press.

Watson, D., \& Clark, L. A. (1994). The PANAS-X: Manual for the positive and negative affect schedule - expanded form. Unpublished manuscript. http://www.psychology.uiowa.edu/faculty/Clark/PANAS-X.pdf.

Watson, D., Clark, L. A., \& Tellegen, A. (1988). Development and validation of brief measures of positive and negative affect: The PANAS scales. Journal of Personality and Social Psychology, 54, 1063-1107.

Watson, D., \& Tellegen, A. (1985). Toward a consensual structure of mood. Psychological Bulletin, 98, 219-235.

Woodworth, R. S., \& Schlosberg, H. (1938/1954). Experimental psychology. New York: Holt, Rinehart, \& Winston. 
APPENDIX 
Table 1

Correlations for Intended Determination Expressions:

Correct and Mis-Identifications

\begin{tabular}{lccccccc}
\hline & correct & misid & misid & misid & misid & misid & misid \\
& ID & anger & joy & neut & sad & disg & fear \\
1. misid anger & $0.37^{*}$ & & & & & & \\
2. misid joy & -0.20 & $-0.39 *$ & & & & \\
3. misid neut & -0.20 & $-0.53^{* *}$ & -0.14 & & & \\
4. misid sadness & -0.11 & 0.18 & $-0.39 *$ & -0.23 & & \\
5. misid disgust & -0.12 & 0.14 & -0.22 & $-0.52^{* *}$ & 0.06 & & \\
6. misid fear & 0.02 & -0.16 & -0.09 & -0.01 & -0.08 & -0.05 & \\
7. misid none & 0.11 & $-0.35^{*}$ & 0.17 & $-0.41^{*}$ & -0.02 & 0.25 & $0.30^{+}$ \\
\hline
\end{tabular}


Percentages of Identifications of Emotion Expressions for Individual Expressers

$\%$ Correct Identifications

\begin{tabular}{|c|c|c|c|c|}
\hline ID & Joy & Anger & Determ & Joy/Anger \\
\hline 1 & $91.58 * * *$ & 33.91 & $58.32 * * *$ & 0.00 \\
\hline 2 & $93.74^{* * *}$ & 28.94 & 9.72 & 0.00 \\
\hline 3 & $98.42 * * *$ & 21.17 & 4.97 & 4.08 \\
\hline 4 & $91.58 * * *$ & 5.18 & 3.02 & 2.56 \\
\hline 5 & $88.77 * * *$ & $48.81^{* * *}$ & $33.05^{* * *}$ & 3.85 \\
\hline 6 & $92.22 * * *$ & 9.50 & $27.21^{* * *}$ & 2.78 \\
\hline 7 & $89.63^{* * *}$ & $66.74 * * *$ & $51.40^{* * *}$ & 0.00 \\
\hline 8 & $89.85^{* * *}$ & $41.47^{* * *}$ & 18.79 & 2.13 \\
\hline 9 & $87.04 * * *$ & 23.54 & 12.74 & 0.00 \\
\hline 10 & $92.01^{* * *}$ & 21.17 & 8.21 & 2.70 \\
\hline 11 & $90.28 * * *$ & 7.99 & $43.84^{* * *}$ & 0.00 \\
\hline 12 & $93.09 * * *$ & $65.01^{* * *}$ & 26.13 & 0.00 \\
\hline 13 & $89.85^{* * *}$ & 15.55 & 16.20 & 2.13 \\
\hline 14 & $91.79 * * *$ & $35.21^{* * *}$ & 26.13 & 0.00 \\
\hline 15 & $90.06 * * *$ & $23.76^{* * *}$ & 6.48 & 2.17 \\
\hline 16 & $93.09 * * *$ & $36.07^{* * *}$ & 4.54 & 0.00 \\
\hline
\end{tabular}

\% Misidentifications Intended Emotion/Identified Emotion

\begin{tabular}{|c|c|c|c|c|}
\hline Joy/Determ & Anger/Joy & Anger/Determ & Determ/Joy & Determ/Anger \\
\hline 7.69 & 0.65 & $54.58 * * *$ & 8.29 & 0.00 \\
\hline 0.00 & 0.00 & 11.25 & 2.15 & 5.74 \\
\hline 8.16 & 0.27 & 14.25 & $68.86^{* * *}$ & 0.23 \\
\hline 2.56 & 2.05 & 20.73 & 0.45 & 1.34 \\
\hline 1.92 & 0.42 & 20.68 & 0.32 & 36.13 \\
\hline 2.78 & 1.43 & 18.62 & 21.66 & 1.48 \\
\hline 4.17 & 16.88 & 14.94 & 2.67 & 38.22 \\
\hline 0.00 & 0.00 & 14.76 & 0.27 & $52.66 * * *$ \\
\hline 5.00 & 2.26 & $50.28 * * *$ & 0.50 & 6.44 \\
\hline 2.70 & 0.55 & 28.77 & 0.24 & 5.41 \\
\hline 2.22 & 0.00 & 25.12 & 0.38 & 32.69 \\
\hline 3.13 & 0.00 & 38.27 & 0.58 & 14.04 \\
\hline 2.13 & 0.77 & $32.74 * * *$ & 9.54 & 1.03 \\
\hline 2.63 & 0.33 & 32.33 & 0.58 & $47.66 * * *$ \\
\hline 0.00 & 0.00 & 9.63 & 1.39 & 3.46 \\
\hline 3.13 & 0.00 & 29.39 & 0.45 & 25.34 \\
\hline
\end{tabular}

*** modal response; $p<.001$ 
Table 2 continued

\% Correct Identifications

\begin{tabular}{|c|c|c|c|}
\hline Joy & Anger & Determ & Joy/Anger \\
\hline $90.28 * * *$ & $57.24^{* * *}$ & $30.02 * * *$ & 2.22 \\
\hline $92.44 * * *$ & 6.91 & 12.10 & 0.00 \\
\hline $90.06 * * *$ & 12.53 & 2.59 & 2.17 \\
\hline $89.63 * * *$ & $84.23 * * *$ & 15.98 & 0.00 \\
\hline $90.06 * * *$ & $40.82 * * *$ & 4.75 & 2.17 \\
\hline $91.36 * * *$ & $61.12^{* * *}$ & 7.78 & 0.00 \\
\hline $91.36 * * *$ & 11.88 & 7.13 & 5.00 \\
\hline $89.42 * * *$ & $36.50^{* * *}$ & $36.50 * * *$ & 2.04 \\
\hline $87.69 * * *$ & 73.65*** & $20.30^{* * *}$ & 1.75 \\
\hline $90.71 * * *$ & $49.46^{* * *}$ & 27.86 & 0.00 \\
\hline $93.52 * * *$ & $69.33 * * *$ & 9.07 & 0.00 \\
\hline $91.58 * * *$ & 11.23 & 13.82 & 2.56 \\
\hline $88.55^{* * * *}$ & $51.62 * * *$ & 15.55 & 1.89 \\
\hline $89.63 * * *$ & $48.16 * * *$ & 4.10 & 2.08 \\
\hline $90.50 * * *$ & 33.91 & 19.87 & 2.27 \\
\hline $88.77 * * *$ & 29.37 & 28.29 & 1.92 \\
\hline $90.93 * * *$ & $40.17^{* * *}$ & 15.12 & 0.00 \\
\hline
\end{tabular}

\% Misidentifications Intended Emotion/Identified Emotion

\begin{tabular}{|c|c|c|c|c|}
\hline Joy/Determ & Anger/Joy & Anger/Determ & Determ/Joy & Determ/Anger \\
\hline 2.22 & 0.51 & 27.78 & 23.15 & 34.57 \\
\hline 2.86 & 9.74 & 31.79 & $55.28 * * *$ & 0.49 \\
\hline 2.17 & 0.49 & 0.25 & 0.44 & 1.77 \\
\hline 4.17 & 0.00 & 30.14 & 19.54 & 0.51 \\
\hline 2.17 & 0.00 & 18.98 & 0.45 & 1.81 \\
\hline 2.50 & 0.00 & 28.89 & 33.96 & 0.23 \\
\hline 2.50 & 6.37 & 10.29 & 20.47 & 0.93 \\
\hline 2.04 & 0.34 & 37.07 & 2.38 & 1.36 \\
\hline 1.75 & 0.82 & 14.75 & 0.27 & $62.06 * * *$ \\
\hline 6.98 & 0.43 & 12.82 & 0.00 & $46.11^{* * *}$ \\
\hline 3.33 & 0.00 & 30.28 & 0.71 & $74.82^{* * *}$ \\
\hline 2.56 & 0.00 & 7.30 & 10.78 & 10.53 \\
\hline 1.89 & 1.34 & 32.59 & 12.53 & 8.95 \\
\hline 2.08 & 0.00 & 13.33 & 0.90 & 4.50 \\
\hline 0.00 & 1.31 & $63.73^{* * *}$ & 26.95 & 4.85 \\
\hline 1.92 & 0.31 & 13.46 & 0.00 & $60.84 * * *$ \\
\hline 4.76 & 0.36 & 26.71 & 19.59 & 1.02 \\
\hline
\end{tabular}

$* * *$ modal response; $p<.001$ 
Table 3

Correlations for Emotion Identifications and Individual Differences Measures

$\begin{array}{cccccccccc}\text { joy } & \text { anger } & \text { determ } & \text { BAS } & \text { BAS } & \text { BAS } & \text { BIS } & \text { phys } & \text { verb } & \\ \text { correct } & \text { correct } & \text { correct } & \text { rew res } & \text { drive } & \text { fun } & & \text { aggr } & \text { aggr } & \text { anger }\end{array}$

\begin{tabular}{|c|c|c|c|c|c|c|c|c|c|c|}
\hline anger correct & -0.16 & & & & & & & & & \\
\hline determ correct & -0.16 & 0.17 & & & & & & & & \\
\hline BAS/rew resp & -0.07 & $0.44 *$ & 0.11 & & & & & & & \\
\hline BAS/drive & -0.26 & $0.30^{+}$ & -0.07 & $0.41 *$ & & & & & & \\
\hline BAS/fun & -0.11 & $0.35 *$ & 0.24 & $0.44 *$ & $0.61^{* *}$ & & & & & \\
\hline BIS & $0.30^{+}$ & $-0.32^{+}$ & -0.20 & 0.08 & $-0.36 *$ & $-0.40^{*}$ & & & & \\
\hline phys aggr & $-0.48 * *$ & 0.11 & $0.32^{+}$ & 0.16 & $0.43^{*}$ & $-0.58 * *$ & $-0.42^{*}$ & & & \\
\hline verb aggr & $-0.33^{+}$ & -0.19 & 0.17 & 0.00 & $0.29^{+}$ & $0.30^{+}$ & $-0.33^{+}$ & $0.68^{* *}$ & & \\
\hline anger & -0.26 & -0.14 & $0.30^{+}$ & 0.12 & 0.19 & 0.20 & 0.14 & $0.66^{* *}$ & $0.56^{* *}$ & \\
\hline hostility & 0.06 & -0.27 & 0.03 & 0.01 & -0.26 & $-0.32^{+}$ & $0.43^{*}$ & 0.10 & 0.28 & $0.57 * *$ \\
\hline
\end{tabular}

${ }^{* *} \mathrm{p}<.01,{ }^{*} \mathrm{p}<.05,{ }^{+} \mathrm{p}<.10$ 


\section{VITA}

Name: $\quad$ Cindy Harmon-Jones

Address: $\quad$ Psychology Department, 4235 TAMU, College Station, TX 77843

Email Address: cindyharmonjones@gmail.com

Education: $\quad$ B.S., Psychology, Excelsior College, 2003 